\title{
Injected Versus Oral Cyclosporine for Human Neural Progenitor Grafting in Rats
}

\author{
Matthew B. Jensen*, Rajeev Krishnaney-Davison, Laura K. Cohen and Su-Chun Zhang \\ Department of Neurology, University of Wisconsin, USA
}

\begin{abstract}
Background: Neural cell transplantation is a promising therapy for stroke, but rejection of human cells in animal models is an obstacle to furthering this research. Many antirejection strategies have been reported, but few comparison data are available. We asked if human neural cell grafts would have different survival or differentiation with injected or oral cyclosporine regimens.

Methods: Rats received intracerebral grafts of human embryonic stem cell-derived neural progenitors, and 6 rats each were randomized to 4 cyclosporine regimens: 1) daily injections, 2) initial injections followed by oral drug in the drinking water, 3) oral drug only, or 4) no cyclosporine. Histology was performed 14 days after grafting for quantification of markers of human cells, neural cell types, and immune cells.

Results: More rats in the injection (6/6) and injection+oral (5/6) groups had surviving graft cells than in the oral $(1 / 6)$ and control $(3 / 6)$ groups $(p<0.05)$, with a trend toward a greater number of surviving graft cells as well. All rats with surviving graft cells also had these cells co-label for a neural progenitor marker, and a minority of graft cells colabeled for a cell division marker and a neuronal marker. Rats with areas of dead graft cell debris were seen in all of the groups. In these areas, cells that labeled for microglial markers also contained the human nuclear marker in their cytoplasm, suggesting phagocytosis of the graft cells.

Conclusions: Human neural cell survival in rat brain tissue differed between cyclosporine regimens, but microglial phagocytosis of graft cells occurred in all the groups. Frequent injection of laboratory animals is undesirable, and a compromise strategy of peritransplant injections followed by drug in the drinking water showed good results in preventing graft cell rejection. Further research is needed to optimize the antirejection approach for this application.
\end{abstract}

Keywords: Cyclosporine; Antirejection; Immunosuppression; Neural progenitor; Embryonic stem cell; Grafting; Transplantation; Human; Rat; Differentiation

\section{Introduction}

\section{Background}

Neural progenitor cell transplantation is a promising therapy to improve outcome for stroke and other neurological disorders, but grafting parameters require further refinement through research in animal models prior to successful translation into human trials [1]. Rats are ideal for research in optimizing transplantation variables for the human neural progenitors that will be used in human trials, but a rat's immune system may reject human cell xenografts [2,3]. Human neural progenitors specifically need to be tested, though, because there may be substantial differences in the behavior of graft cells derived from different species that may affect their safety or efficacy $[2,3]$.

We recently performed a systematic review of human neural cell transplantation in animal stroke models, and found wide variability in the antirejection strategy used by different investigators, with only half reporting the use of any antirejection drugs [4]. The most common regimen reported was cyclosporine alone, with doses ranging from five to $20 \mathrm{mg} / \mathrm{kg}$; multiple routes of administration including oral, subcutaneous, intraperitoneal, and intramuscular; and varying schedules including daily, every other day, or three times a week. All of these publications reported graft cell survival of variable or unquantified amounts, and no group reported clinical adverse events related to the antirejection drugs. Only one study reported a direct comparison of an antirejection strategy (cyclosporine) and an identically treated group that was not given an antirejection drug, where they found better graft cell survival and improved recovery of stroke deficits in the cyclosporine group [5]. We did not find reports of direct comparisons of different active antirejection strategies in our review. A subsequent article has reported similar graft cell survival with daily cyclosporine injections for two weeks when compared to longer-term daily injections [6].

Most of the reported regimens used the antirejection drug cyclosporine administered by daily injection. Long-term frequent injection of laboratory animals is labor-intensive, painful for the animals, and may also confound functional analysis by inducing behavioral changes. We, therefore, sought to evaluate the effects of injected versus oral cyclosporine regimens on human neural cell graft outcomes and rat brain tissue responses to identify a strategy that may be amenable for future long-term graft survival.

\section{Methods}

The human embryonic stem cell line H9 (WiCell) was expanded, directed to a neural progenitor cell identity, and prepared for grafting as previously described [7]. On differentiation day 21 , we prepared the

*Corresponding author: Matthew B. Jensen, MD, 1685 Highland Ave, room 7273 Madison, WI 53705, Tel: 608-263-5420; Fax: 608-263-0412; E- Mail: jensen@ neurology.wisc.edu

Received April 04, 2012; Accepted April 18, 2012; Published April 20, 2012

Citation: Jensen MB, Krishnaney-Davison R, Cohen LK, Zhang SC (2012) Injected Versus Oral Cyclosporine for Human Neural Progenitor Grafting in Rats. J Stem Cell Res Ther S10:003. doi:10.4172/2157-7633.S10-003

Copyright: (c) 2012 Jensen MB, et al. This is an open-access article distributed under the terms of the Creative Commons Attribution License, which permits unrestricted use, distribution, and reproduction in any medium, provided the original author and source are credited. 
cells for grafting with 50,000 cells/ul in artificial cerebrospinal fluid (Harvard Apparatus) with 1:10,000 Rho Kinase inhibitor (Tocris).

The animal care and use committee of the University of Wisconsin approved all animal procedures, and the results of all the rats used are presented below. Male 8-week-old Sprague Dawley rats (Charles River) were anesthetized with isoflurane and administered bupivicaine and buprenorphine for analgesia. A small craniotomy was made $3 \mathrm{~mm}$ to the left of bregma. A drawn glass needle was slowly inserted to a depth of $6 \mathrm{~mm}$ from the skull and left in place for $3 \mathrm{~min}$ prior to the first injection. The cell suspension was injected with $1 \mathrm{ul}$ boluses over 1 minute each at $1 \mathrm{~mm}$ increments from 6 to $2 \mathrm{~mm}$ deep to the skull, for a total of 250,000 cells grafted, and the needle was left in place for 3 minutes prior to removal. This injection method created long, linear grafts maximizing graft cell exposure to the host tissue.

Each rat was randomized to 1 of 4 cyclosporine regimens, with 6 rats per group. The injection group received cyclosporine (Neoral, Novartis) $10 \mathrm{mg} / \mathrm{kg} /$ day subcutaneously from 2 days prior to grafting until perfusion. The injection+oral group received cyclosporine 10 $\mathrm{mg} / \mathrm{kg} /$ day subcutaneously for 7 days starting 2 days prior to grafting, followed by $100 \mathrm{ug} / \mathrm{ml}$ in the drinking water until perfusion. The oral group received cyclosporine $100 \mathrm{ug} / \mathrm{ml}$ in the drinking water starting 2 days prior to grafting until perfusion. The control group received no cyclosporine.

The rats were perfused and brain sections prepared as previously described 14 days after grafting [7]. Immunohistochemistry was performed with standard procedures using primary antibodies for the following markers: human-specific nuclei (HUNU, 1:200, Millipore), human-specific cytoplasm (STEM121, 1:500, StemCells), humanspecific neural progenitors (nestin, 1:3000, Millipore), neurons (MAP2, 1:1000, Millipore), astrocytes (GFAP, 1:5000, DAKO), dividing cells (KI67, 1:500, Invitrogen), leukocytes (CD45, 1:500, Santa Cruz), neutrophils (MPO, 1:1000, Fisher), T lymphocytes (CD3, 1:30, BD Biosciences), and microglia (CD11B, 1:1000, Santa Cruz). Hoechst (Invitrogen) was used to label all nuclei. As previously described, the number of labeled cells was stereologically estimated on visible grafts, co-labeling of cells was confirmed and quantified with confocal microscopy, and optical densitometry of the integrated density was determined for fluorescent markers [7].

Data are presented as proportions or means \pm standard deviation (SD). Fisher's exact test was used to assess for the primary outcome of group differences in the proportion of rats with surviving or dead grafts. The paired t-test was used to compare marker integrated density values of the grafted hemispheres to the contralateral hemispheres. One-way ANOVA was used to assess for group differences in the mean number of stereologically estimated labeled cells, graft cells co-labeled for secondary markers, and marker integrated density values. Tukey's post-hoc test was used to determine which groups differed.

\section{Results}

No clinical adverse events related to the grafts or the cyclosporine were seen during the survival period; specifically, we did not see clinical signs of tumors, seizures, or infections in any of the rats. There was a significant difference in the primary outcome of the proportion of rats with surviving graft cells between the 4 groups $(\mathrm{p}=0.017)$. The combined injection and injection+oral groups had significantly more rats with surviving graft cells $(11 / 12)$ than the combined oral and control groups $(4 / 12, p=0.009$, Figure 1$)$. The mean number of surviving graft cells in the injection+oral group was larger than the other 3 groups combined, both when all of the rats of each group were included or when just the rats with surviving graft cells were included, but this difference was not statistically significant due to high variability ( $p>0.05$, Table 1$)$.

All rats with surviving graft cells had these cells co-label with the neural progenitor marker nestin (Figure 2). No rats had graft cells colabel for the astrocyte marker GFAP. Small proportions of graft cells co-labeled for the cell division marker KI67 and the neuronal marker MAP2, but MAP2 staining was weak when present (Figure 2). There were no significant differences for the presence or proportions of graft cells co-labeled for these markers between the 4 groups ( $p>0.05$, Table 2). No tumors were seen in the brain sections.

Optical densitometry demonstrated significantly more overall cellularity (Hoechst integrated density of $2.21 \times 10^{8}$ versus $2.01 \times 10^{7}$, $\mathrm{p}=0.007$ ), significantly more reactive astrocytosis (GFAP integrated

\begin{tabular}{|c|c|c|c|c|c|}
\hline Group & $\begin{array}{l}\text { Rats with live graft cells } \\
(\%)\end{array}$ & $\begin{array}{l}\text { Rats with dead graft cell } \\
\text { debris }(\%)\end{array}$ & $\begin{array}{l}\text { Rats with both live graft } \\
\text { cells and dead graft cell } \\
\text { debris }(\%)\end{array}$ & $\begin{array}{l}\text { Mean live graft cells }( \pm S D) \\
\text { for all rats }\end{array}$ & $\begin{array}{l}\text { Mean live graft cells }( \pm S D) \\
\text { excluding rats without live } \\
\text { graft cells }\end{array}$ \\
\hline Injection & $6 / 6(100 \%)$ & $1 / 6(17 \%)$ & $1 / 6(17 \%)$ & $141,836( \pm 268,273)$ & $141,836( \pm 268,273)$ \\
\hline Injection+oral & $5 / 6(83 \%)$ & $3 / 6(50 \%)$ & $2 / 6(33 \%)$ & $347,857( \pm 625,700)$ & $417,428( \pm 673,108)$ \\
\hline Oral & $1 / 6(17 \%)$ & $2 / 6(33 \%)$ & $0 / 6(0 \%)$ & $1,595( \pm 3,615)$ & $4,784( \pm 5,901)$ \\
\hline Control & $3 / 6(50 \%)$ & $3 / 6(50 \%)$ & $1 / 6(17 \%)$ & $113,733( \pm 250,056)$ & $227,467( \pm 342,802)$ \\
\hline
\end{tabular}

Table 1: Graft cell survival.

\begin{tabular}{|l|l|l|l|l|}
\hline \multirow{2}{*}{ Group } & Percent of graft cells that co-labeled for these markers $($ mean $\% \pm S D)$ & \multicolumn{2}{l|}{} \\
\cline { 2 - 4 } & Nestin & MAP2 & GFAP \\
\hline Injection & $74 \%( \pm 38)$ & $19 \%( \pm 40)$ & $0 \%$ & $0 \%$ \\
\hline Injection+oral & $56 \%( \pm 43)$ & $12 \%( \pm 20)$ & $0 \%$ & $18 \%( \pm 11)$ \\
\hline Oral & $87 \%$ & $0 \%$ & $0 \%$ & $23 \%$ \\
\hline Control & $48 \%( \pm 50)$ & $0 \%$ & $9 \%( \pm 8)$ \\
\hline
\end{tabular}

Table 2: Graft cell differentiation. 


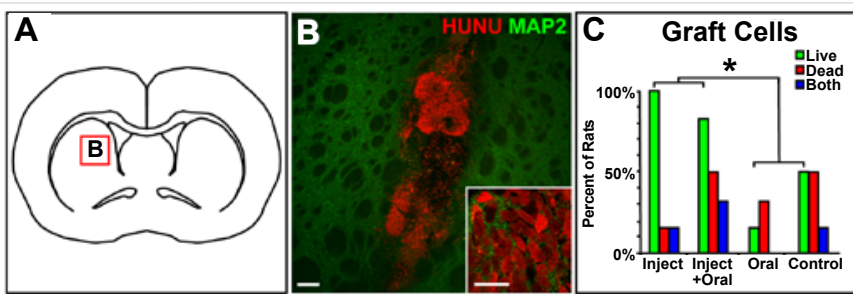

Figure 1: Panel $\mathrm{A}$ is an illustration of a coronal rat brain section at bregma, and the red box in the striatum indicates the location of grafts in panel $B$ and the subsequent panels. Panel B shows live graft cells in rat striatum with the intact human nuclei (HUNU) of the graft cells labeled red and rat or human neuronal MAP2 labeled green (scale bar $=100 \mathrm{um}, 10 \mathrm{um}$ for the inset). Panel C shows the percent of rats in each experimental group with live graft cells (green bars), dead graft cell debris (red bars), or rats that had both live graft cells and dead graft cell debris (blue bars); * indicates $p<0.05$.
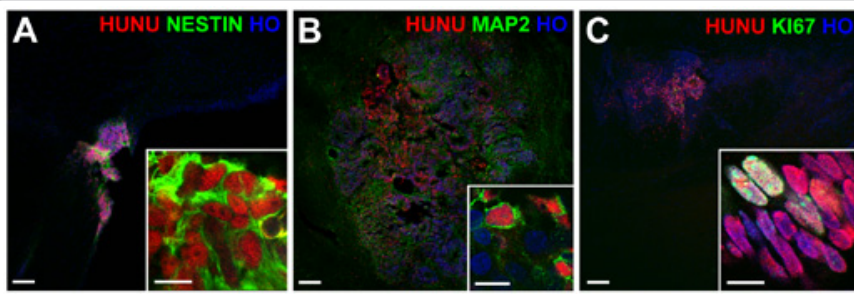

Figure 2: Rat brain sections with live graft cells. The red-labeled human nuclei (HUNU) of graft cells also co-labeled green for the neural progenitor marker nestin (A), and, to a lesser extent, the neuronal marker MAP2 (B) and the marker of dividing cells KI67 (C). All nuclei are labeled blue with Hoechst (HO); scale bars $=100 \mathrm{um}$ (10 um for the insets).
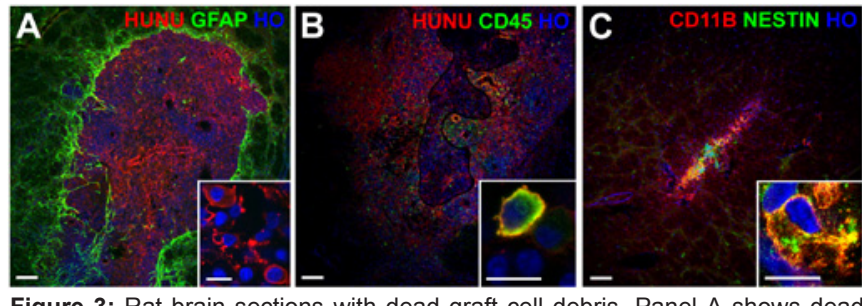

Figure 3: Rat brain sections with dead graft cell debris. Panel A shows dead graft cell debris with the red-labeled human nuclear marker (HUNU) surrounded by reactive astrocytes labeled green for GFAP. The inset of panel A shows that the HUNU marker, which should only be in the nuclei of intact graft cells, was instead found in the cytoplasm of cells invading the graft area with a morphology consistent with activated microglia. Panels $B$ and $C$ show grafts with areas of live graft cells with intact nuclei as well as areas of dead graft cell debris. Panel $B$ shows cells with the morphology of activated microglia having cytoplasm that co-labeled for HUNU in red and the leukocyte marker CD45 in green. Panel C shows cells with cytoplasm that co-labeled for the microglial marker CD11B in red and a human-specific antibody for the neural progenitor marker nestin in green. All nuclei are labeled blue with Hoechst (HO); scale bars = 100 um (10 um for the insets).

density of $5.07 \times 10^{8}$ versus $\left.2.60 \times 10^{8} \mathrm{p}=0.004\right)$, and a trend toward increased microglia (CD11B integrated density of $4.72 \times 10^{8}$ versus 3.04 $\left.\mathrm{x} 10^{8}, \mathrm{p}=0.08\right)$ in the grafted hemispheres compared to the contralateral hemispheres, but no significant differences were seen between the 4 groups ( $>0.05$, Figure 3 ). Areas that appeared to be dead graft cell debris were seen in some rats of all 4 groups, both with and without intermixed intact surviving graft cells (Figure 3). There was no significant difference in the proportion of rats with dead graft cell debris between the 4 groups ( $p>0.05$, Table 1 ). Immunostaining of areas with dead graft cell debris showed the presence of the human-specific nuclear marker HUNU in the cytoplasm of cells with morphology consistent with activated microglia. These cells also labeled for the leukocyte marker CD45 and the microglia marker CD11B, suggesting that the expected mechanism of cerebral graft cell rejection had occurred through microglial phagocytosis of the graft cells (Figure 3). Only rare cells in just a few of the rats labeled for the neutrophil marker MPO or the T lymphocyte marker CD3.

\section{Discussion}

We found differences in the survival of human neural cells transplanted into the brains of rats administered different cyclosporine regimens over a short survival period, but also evidence of microglial phagocytosis of graft cells and reactive astrocytosis in all the groups. Laboratories investigating the use of human neural cell grafts to improve recovery of rat models of stroke and other neurological disorders would benefit from a simple and minimally-invasive antirejection strategy for the xenograft cells, as progressively longer survival times will be needed to evaluate durability of graft benefit over time. In particular, neural progenitor cells may require months to fully mature and integrate into injured host brain networks, depending on the maturation state of the cells at the time of transplantation [8]. Frequent injections are undesirable for both laboratory animals and personnel, and oral administration of drugs mixed in the drinking water would be ideal if it were similarly effective. Unfortunately, we did not see equivalent graft cell survival with oral cyclosporine alone, or when no cyclosporine was given. We did, however, have good success with a compromise strategy of peritransplant injections followed by mixing the drug in the drinking water. This regimen produced a similar proportion of rats having surviving grafts when compared to the injection-only group, and a trend towards a greater number of surviving graft cells. We have previously demonstrated good survival of human neural progenitor cells using this strategy for rats transplanted one week after cerebral infarction followed by one month of survival time after grafting [7].

Despite good graft cell survival in several of our experimental groups, in all the groups at least some of the rats lost part or all of their grafts. This appeared to have occurred through host tissue immune rejection, based on the microglial and astrocytic responses we found in and around the grafts, as well as the evidence of microglial phagocytosis of graft cells. Our primary finding of differences in graft cell survival between the different cyclosporine regimens also suggests that the mechanism of graft cell loss is via host immune rejection, as the primary effect of this drug is prevention of graft tissue rejection by the immune system.

Limitations of our study include the focused nature of the antirejection strategies we tested, and the relatively short survival period evaluated after grafting, although we did find differences between the groups during this time interval. Further research beyond the scope of our study is clearly needed to optimize the antirejection approach for human neural cell grafting, including studies of larger groups followed for longer survival periods using cyclosporine at multiple doses, routes, and schedules, as well as factorial evaluations of multidrug combinations including cyclosporine and the numerous other antirejection drugs that are currently used by patients after organ transplantation.

\section{Conclusions}

We found that peritransplant injections of cyclosporine appear to be necessary to prevent rejection of human neural cell grafts in rat brain tissue, and also that the injections may be switched to oral administration by mixing the drug in the drinking water after the peritransplant period. For future directions, we recommend that 
Citation: Jensen MB, Krishnaney-Davison R, Cohen LK, Zhang SC (2012) Injected Versus Oral Cyclosporine for Human Neural Progenitor Grafting in Rats. J Stem Cell Res Ther S10:003. doi:10.4172/2157-7633.S10-003

Page 4 of 4

additional single and multidrug antirejection strategies be directly compared to determine if there is a superior approach to the one described here.

\section{Acknowledgement}

This publication was funded by NIH grants 9U54TR000021and P30HD03352.

\section{References}

1. Bliss TM, Andres RH, Steinberg GK (2010) Optimizing the success of cell transplantation therapy for stroke. Neurobiol Dis 37: 275-283.

2. Stem Cell Therapies as an Emerging Paradigm in Stroke Participants (2009) Stem Cell Therapies as an Emerging Paradigm in Stroke (STEPS): Bridging Basic and Clinical Science for Cellular and Neurogenic Factor Therapy in Treating Stroke. Stroke 40: 510-515

3. Savitz SI, Chopp M, Deans R, Carmichael ST, Phinney D, et al. (2011) Stem Cell Therapy as an Emerging Paradigm for Stroke (STEPS) II. Stroke 42: 825829.

4. Jensen MB, Al Sawaf A, Zhang SC (2011) Human Neural Stem Cell
Transplantation in Animal Stroke Models: A Systematic Review. European Neurological Journal, in press.

5. Borlongan CV, Tajima Y, Trojanowski JQ, Lee VM, Sanberg PR (1998) Transplantation of cryopreserved human embryonal carcinoma-derived neurons (NT2N cells) promotes functional recovery in ischemic rats. Exp Neurol 149: 310-321.

6. Rota Nodari L, Ferrari D, Giani F, Bossi M, Rodriguez-Menendez V, et al. (2010) Long-term survival of human neural stem cells in the ischemic rat brain upon transient immunosuppression. PLoS One 5: e14035.

7. Jensen MB, Yan H, Krishnaney-Davison R, Al Sawaf A, Zhang SC (2011) Survival and Differentiation of Transplanted Neural Stem Cells Derived from Human Induced Pluripotent Stem Cells in a Rat Stroke Model. J Stroke Cerebrovasc Dis

8. Johnson MA, Weick JP, Pearce RA, Zhang SC (2007) Functional neura development from human embryonic stem cells: accelerated synaptic activity via astrocyte coculture. J Neurosci 27: 3069-3077.
This article was originally published in a special issue, Embryonic and Induced Pluripotent Stem Cells handled by Editor(s). Dr. Jianlong Wang, Mount Sinai School of Medicine, United States 